\title{
Dopamine Supports Coupling of Attention-Related Networks
}

\author{
Linh C. Dang, ${ }^{1,2}$ James P. 0'Neil, ${ }^{2}$ and William J. Jagust ${ }^{1,2}$ \\ ${ }^{1}$ Helen Wills Neuroscience Institute, University of California, Berkeley, Berkeley, California 94720-3190, and ${ }^{2}$ Lawrence Berkeley National Laboratory, \\ Berkeley, California 94720-8119
}

\begin{abstract}
Attentional processing has been associated with the dorsal attention, default mode, and frontoparietal control networks. The dorsal attention network is involved in externally focused attention whereas the default mode network is involved in internally directed attention. The frontoparietal control network has been proposed to mediate the transition between external and internal attention by coupling its activity to either the dorsal attention network or the default mode network, depending on the attentional demand. Dopamine is hypothesized to modulate attention and has been linked to the integrity of these three attention-related networks. We used PET with 6-[ $\left.{ }^{18} \mathrm{~F}\right]$ fluoro-L-m-tyrosine to quantify dopamine synthesis capacity in vivo and fMRI to acquire stimulus-independent brain activity in cognitively healthy human subjects. We found that in the resting state where internal cognition dominates, dopamine enhances the coupling between the frontoparietal control network and the default mode network while reducing the coupling between the frontoparietal control network and the dorsal attention network. These results add a neurochemical perspective to the role of network interaction in modulating attention.
\end{abstract}

\section{Introduction}

fMRI studies of attention revealed the importance of three brain networks: a dorsal attention network (DAN), a default mode network (DMN), and a frontoparietal control network (FPCN). The DAN is hypothesized to modulate externally directed attention by amplifying or attenuating the saliency of relevant and irrelevant cues (Corbetta and Shulman, 2002; Ptak and Schnider, 2010). This network includes the intraparietal sulcus, frontal eye fields, inferior parietal lobule, dorsolateral prefrontal cortex, insula, and supplementary motor and middle temporal areas (Fox et al., 2005). The DMN, which includes the posterior cingulate, precuneus, medial prefrontal, and lateral parietal and medial temporal cortices, is associated with internal cognitive processes and deactivates when attention is focused on the external environment (Raichle et al., 2001; Greicius et al., 2003). Analysis of resting state $\mathrm{fMRI}$ signal revealed that activities in the DAN and DMN are anticorrelated (Fox et al., 2005). Task-related fMRI showed that goal-directed behavior is associated with increased DAN and decreased DMN activities whereas stimulus-independent thoughts are associated with increased DMN and decreased DAN activities (Corbetta et al., 2002; McKiernan et al., 2003; Raichle and Snyder, 2007). These results led to the proposal that competition between these two networks underlies the transition between taskfocused attention and stimulus-independent thought.

Mediating the allocation of resources between the DAN and the DMN may be the FPCN, which encompasses the anterior inferior parietal lobule, anterior insular cortex, and anterior prefrontal, dorsolateral prefrontal, and dorsomedial superior fron-

\footnotetext{
Received Feb. 22, 2012; revised May 23, 2012; accepted May 28, 2012.

Author contributions: L.C.D. and W.J. designed research; L.C.D., J.P.O., and W.J. performed research; L.C.D. analyzed data; L.C.D. and W.J. wrote the paper.

This study was supported by a grant from the National Institute on Aging (AG027984).

Correspondence should be addressed to Linh C. Dang, Helen Wills Neuroscience Institute, 132 Barker Hall 3190, Berkeley, CA 94720-3190. E-mail: linhdang@berkeley.edu.

DOI:10.1523/JNEUROSCI.0909-12.2012

Copyright $\odot 2012$ the authors $\quad 0270-6474 / 12 / 329582-06 \$ 15.00 / 0$
}

tal/anterior cingulate. The FPCN is engaged by task paradigms assessing conflict monitoring, planning, and reasoning (Kroger et al., 2002; van den Heuvel et al., 2003; Wager et al., 2004). Vincent and colleagues (2008) noticed that the FPCN is physically interposed between the DAN and the DMN and suggested that the FPCN may flexibly couple to the DMN or the DAN depending on the attentional demand of the task. Spreng and colleagues (2010) provided support for this proposal by showing that the FPCN is coupled to the DMN during internal cognition and uncoupled during external cognition.

Also connecting the DAN, DMN, and FPCN is their mutual association with the neurotransmitter dopamine. Methylphenidate, which blocks dopamine reuptake, increases DAN activation during performance of visual attention and memory tasks (Müller et al., 2005; Tomasi et al., 2011). Dopamine agonists also influence DMN deactivation and alter the relationship between DMN activity and performance (Nagano-Saito et al., 2009; Tomasi et al., 2011). Activity in the FPCN is influenced by functional polymorphisms in the dopamine system (Tan et al., 2007; Gordon et al., 2011), and dopamine depletion impairs FPCN function and its association with performance (Nagano-Saito et al., 2008).

Given the influence of dopamine on these three networks, we hypothesized that dopamine modulates the cross-network interactions that other researchers have identified. Specifically, without external stimulation, dopamine would influence the degree of correlation between the FPCN and the DMN and anticorrelation between the FPCN and the DAN. We used PET with 6 - $\left[{ }^{18} \mathrm{~F}\right]$ fluoro-L-m-tyrosine (FMT) to measure dopamine synthesis capacity in vivo and fMRI to acquire stimulus-independent brain activity.

\section{Materials and Methods}

Subjects

Twenty-eight healthy, right-handed subjects between 20 and 30 years old, inclusive (mean age: $24.3 \pm 3.0$ years, 12 male, 16 female) partic- 
ipated in the study. PET data from 12 subjects in this study were reported in two previous studies on different topics (Braskie et al., $2008,2011)$. Subjects were recruited via flyers and online postings. Subjects were excluded if they had a Mini Mental State Exam (Folstein et al., 1975) score $<28$, a known major systemic disease, a history of psychiatric or neurological disorder, a history of substance abuse, current usage of medication known to affect dopaminergic or any neurological function, current or prior symptoms of depression, a serious head injury, or any contraindications to MR imaging. All subjects gave written informed consent before participating in the study, which included a PET-FMT scan, a resting state fMRI scan, and structural MRI scans. The current study was approved by institutional review boards at University of California, Berkeley and Lawrence Berkeley National Laboratory.

\section{PET data acquisition}

PET imaging and FMT synthesis were performed at Lawrence Berkeley National Laboratory as described previously (VanBrocklin et al., 2004; Braskie et al., 2008) and briefly summarized below. FMT is a substrate of aromatic L-amino acid decarboxylase, and signal intensity on PETFMT scans is indicative of dopamine synthesis capacity (Jordan et al., 1997). Subjects were given an oral dose of carbidopa $(2.5 \mathrm{mg} / \mathrm{kg}) \sim 60$ min before FMT injection to inhibit peripheral decarboxylation of FMT. PET scans were acquired on a Siemens ECAT-HR PET camera in 3D mode. Approximately $2.5 \mathrm{mCi}$ of high specific activity FMT were injected as a bolus into an antecubital vein followed by $89 \mathrm{~min}$ of dynamic data acquisition. FMT images were reconstructed with an ordered subset expectation maximization algorithm with weighted attenuation, scatter corrected, and smoothed with a $4 \mathrm{~mm}$ full-width at half-maximum kernel.

\section{MRI data acquisition}

MRI was performed on a Siemens 1.5T Magnetom Avanto System with a 12-channel head coil. $A$ foam cushion was used to restrict head movement, and headphones were provided to reduce effects of scanner noise. Resting state fMRI data were acquired with 250 wholebrain, $2^{*}$-weighted echo planar images (repetition time, $1890 \mathrm{~ms}$; echo time, $50 \mathrm{~ms}$; flip angle, $90^{\circ}$; voxel dimensions, $3 \times 3 \times 3.5 \mathrm{~mm}$ ). Subjects were instructed to remain still, relax, and think of nothing in particular. For registration purposes, three structural images were collected for each subject: one T1-weighted image in plane with the fMRI data (repetition time, $2000 \mathrm{~ms}$; echo time, $11 \mathrm{~ms}$; flip angle, $150^{\circ}$; voxel dimensions, $\left.0.9 \times 0.9 \times 3.5 \mathrm{~mm}\right)$ and two T1-weighted volumetric magnetization prepared rapid gradient echo (MPRAGE) images (repetition time, $2120 \mathrm{~ms}$; echo time, $3.58 \mathrm{~ms}$; inversion time, $1100 \mathrm{~ms}$; flip angle, $15^{\circ}$; voxel dimensions, $1 \times 1 \times 1 \mathrm{~mm}$ ). The two MPRAGEs were averaged to obtain a high-resolution structural image. The T1-weighted structural image in-plane with the resting-state fMRI data was used to improve coregistration of resting-state fMRI data to the mean MPRAGE, which was used to normalize resting-state fMRI data to standard MNI space for group analysis.

\section{PET data analysis}

To correct for motion during scanning, all FMT frames were realigned to the twelfth frame using SPM8 (www.fil.ion.ucl.ac.uk/spm/). The MPRAGE with its associated cerebellum and midbrain regions of interest (ROIs) were coregistered to the mean image of all realigned frames in the FMT scan using FSL-FLIRT (http://www.fmrib.ox.ac.uk/fsl/, version 4.1.2). An in-house graphical analysis program implementing Patlak plotting (Patlak and Blasberg, 1985) with the cerebellum as the reference region was used to create $K_{i}$ images, which represent the amount of tracer accumulated in the brain relative to the cerebellum. These images are comparable to $K_{i}$ images obtained using a blood input function but are scaled to the volume of distribution of the tracer in the cerebellar reference region. $K_{i}$ values from the midbrain were extracted.

\section{Regions of interest}

The midbrain and cerebellum were manually drawn on each subject's mean MPRAGE using FSLview (Fig. 1). The midbrain was drawn by visual inspection on five consecutive axial slices, the most caudal being

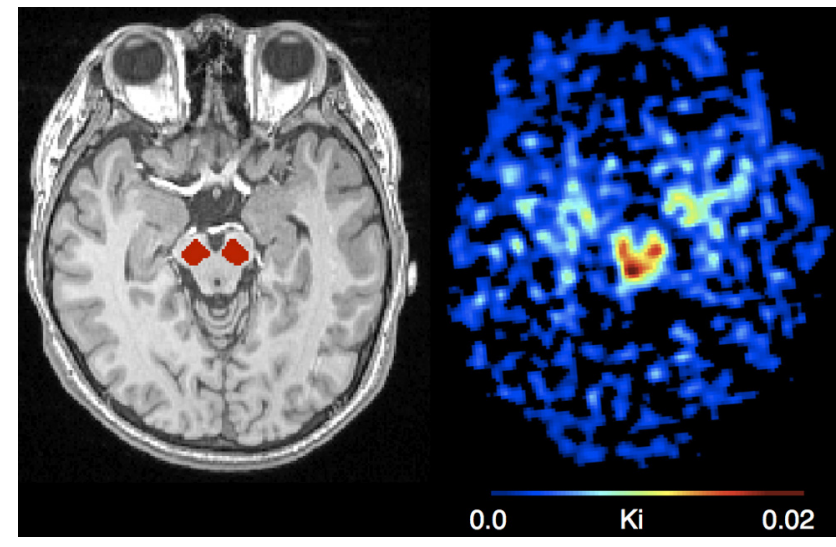

Figure 1. One subject's scans. Left, Midbrain ROls superimposed on a structural scan. Right, FMT uptake in the midbrain.

the slice on which the frontopontine fibers were separated into left and right bundles and the substantia nigra was clearly outlined. Two raters performed the reliability test. One rater drew ROIs more than five times for multiple subjects to achieve intrarater reliability. Both interrater and intrarater reliability were $>95 \%$. The cerebellum was the reference region for calculating FMT signal intensity. Only gray matter was included in the cerebellum ROI. Dopamine-producing neurons are located in the midbrain, which is anterior to the cerebellum. To avoid contamination of FMT signal from the dopaminergic nuclei, only the posterior threefourths of the cerebellum was included in the ROI. After coregistration to FMT-PET space, we thresholded the image intensity within the ROIs at 0.5 to ensure high gray matter probability.

\section{MRI data analysis}

Network identification. We extracted the DAN, DMN, and FPCN using seeds from previous publications. Fox and colleagues (2005) used seeds at the intraparietal sulcus $(-25,-57,-46)$, the frontal eye field $(25,-13,50)$, and the middle temporal region $(-45,-69,-2)$ to generate the DAN. Greicius and colleagues (2003) used the posterior cingulate to define the DMN; the posterior cingulate voxel showing peak functional connectivity in their network had coordinates -2 , $-51,27$. Vincent and colleagues (2008) used left and right dorsolateral prefrontal cortex seeds to extract the FPCN; in their network, peak functional connectivities for left and right seeds had coordinates $-50,20,34$ and $46,14,43$, respectively.

We generated an $8 \times 8 \times 8 \mathrm{~mm}^{3}$ ROI around each of the coordinates above and coregistered the ROIs to individual subject's restingstate fMRI scan. Resting-state fMRI scans were preprocessed in FSL using the following operations: motion correction (Jenkinson et al., 2002), removal of nonbrain matter (Smith, 2002), spatial smoothing with a Gaussian kernel of $5 \mathrm{~mm}$, and high-pass temporal filtering removing frequencies $<0.01 \mathrm{~Hz}$. For each ROI, for each subject, we extracted the mean time series for voxels in the ROI and performed a regression in FSL-FEAT with the mean time series and three nuisance covariates discussed below. $z$-Statistic maps from the regressions were averaged across subjects to generate group-level maps showing correlations between each voxel's time series and the mean time series for each ROI. Group-level maps for ROIs in the intraparietal sulcus, frontal eye field, and middle temporal region were averaged to create the DAN. Since the DMN was defined solely with a posterior cingulate seed, the group-level $z$-statistic map for the posterior cingulate seed was the DMN. The FPCN was the average of group-level maps from left and right prefrontal cortex ROIs.

Nuisance variables. To control for effects of scanner artifacts and physiological processes such as respiratory and cardiac functions, signal associated with white matter, CSF, and the global signal were entered as covariates in the subject-level regression. To extract signal associated with white matter and CSF, each subject's mean MPRAGE was segmented into gray matter, white matter, and CSF using FSL-FAST. Signal 
intensity in CSF and white matter images was then thresholded at $90 \%$ and $99 \%$, respectively, to ensure high tissue probability. Each individual's resting-state fMRI scan was masked with the thresholded CSF and white matter images, and the mean time series was extracted for CSF and white matter. Global signal was calculated by averaging across all voxels in the whole brain.

The removal of the global signal served two purposes in this study. The first purpose was to control for global effects common to all fMRI studies, such as scanner artifacts, gross body movement, and physiological processes. The second purpose was to control for effects of peripheral dopamine activity. Dopamine receptors are present in peripheral arteries, carotid bodies, and the endocrine system. Stimulation of these receptors has been found to affect vasodilation and myocardial contractility (Clark and Menninger, 1980; Cavero et al., 1982; Kohli, 1990). The global signal was removed to remove effects of peripheral dopamine from effects in the CNS.

Cross-network connectivity. From the three networks we extracted, we identified the nodes in each network by first eroding voxels with low intensities to remove voxels that overlapped between nodes and then applying a cluster algorithm in Scipy (www.scipy.org) to identify individual clusters of voxels. We also referenced previous studies to ensure that the clusters in our networks resembled published findings (Greicius et al., 2003; Fox et al., 2005; Vincent et al., 2008). We identified the voxel with the highest $z$-statistic in each cluster or node in the network and created an $8 \times 8 \times 8$ $\mathrm{mm}^{3}$ ROI around the peak voxel. For each node in each network, we extracted the mean time series from the resting-state fMRI data. To calculate how activity in one network is related to activity in one of the other two networks, we calculated Pearson's correlations between the mean time series of each node in each network with the time series of each node in the other network. Since the objective was to understand, within each subject, how different networks relate to one another, we standardized all the Pearson's correlations for each subject to derive a relative measure of coupling; thus, a negative correlation value now means that the strength of coupling is less than the subject's average correlation, not that the coupling is anticorrelated. We then averaged the cross-network Pearson's correlations to form a coupling value for any two networks. For example, the DMN has five nodes and the FPCN has five nodes. We calculated Pearson's correlations between each of five nodes in the DMN with each of five nodes in the FPCN and averaged the resulting 25 Pearson's correlations to derive a measure of how activity in the DMN is correlated with activity in the FPCN.

Statistics. Statistical tests were performed using R (http://www.r-project.org/). We used Pearson's correlations to compare cross-network coupling values with midbrain FMT $K_{i}$ values.

\section{Results}

Figure 2 shows the DAN, DMN, and FPCN extracted with the seed-based approach. Table 1 lists the coordinates and intensity of the peak voxel in each cluster of each network. Before standardization, the time series of every node was positively correlated with the time series of essentially every other node. Average Pearson's correlations were $0.30 \pm 0.13$ for FPCN-DAN cou- pling, $0.30 \pm 0.11$ for FPCN-DMN coupling, and $0.21 \pm 0.15$ for DAN-DMN coupling.

FMT $K_{i}$ values in the midbrain (mean $0.010 \pm 0.002$ ) correlated positively with coupling between the DMN and FPCN $(r=$ $0.38, p$ value $=0.046$ ) but negatively with coupling between the DAN and the FPCN $(r=-0.39, p$ value $=0.038)$. No significant correlation existed between midbrain FMT $K_{i}$ values and coupling between the DAN and the DMN $(r=-0.17, p$ value $=$ 0.395; Fig. 3).

\section{Discussion}

Previous findings suggest that externally focused attention involves the DAN whereas internally directed attention involves the DMN, and the FPCN supports the transition between external and internal attention by coupling its activity with either the DAN or the DMN during externally or internally driven tasks, respectively (Spreng et al., 2010). Here we show that dopamine is involved in this interaction between the three networks. Specifically, in the resting condition, when internal cognitive processes presumably dominate and the DMN is engaged and the DAN is not active, we found that dopamine synthesis capacity correlates positively with the correlation between activity in the DMN and activity in the FPCN and negatively with the coupling between 
Table 1. MNI coordinates and maximum intensity of the peak voxel in each node in the DAN, DMN, and FPCN

\begin{tabular}{|c|c|c|c|c|}
\hline & \multicolumn{3}{|c|}{ MNI coordinates } & \multirow[b]{2}{*}{$\operatorname{Max} z$} \\
\hline & $x$ & $y$ & $z$ & \\
\hline \multicolumn{5}{|l|}{ Dorsal attention network } \\
\hline Precentral area, left & -26 & -2 & 54 & 3.06 \\
\hline Precentral area, right & 28 & -12 & 50 & 4.20 \\
\hline Superior parietal lobule, left & -28 & -54 & 58 & 4.38 \\
\hline Superior parietal lobule, right & 30 & -56 & 50 & 4.32 \\
\hline Middle temporal area, left & -44 & -66 & -4 & 4.22 \\
\hline \multicolumn{5}{|l|}{ Default mode network } \\
\hline Posterior cingulate/precuneus & 0 & -54 & 28 & 10.08 \\
\hline Medial prefrontal & 2 & 62 & 22 & 6.85 \\
\hline Lateral parietal, left & -46 & -64 & 32 & 7.44 \\
\hline Lateral parietal, right & 52 & -64 & 34 & 7.68 \\
\hline Middle temporal, left & -62 & -10 & -16 & 5.09 \\
\hline \multicolumn{5}{|l|}{ Fronto-parietal control network } \\
\hline Dorsomedial frontal/anterior cingulate & -4 & 38 & 38 & 5.59 \\
\hline Dorsolateral prefrontal, right & 50 & 16 & 42 & 7.45 \\
\hline Dorsolateral prefrontal, left & -50 & 14 & 36 & 6.98 \\
\hline Lateral parietal, right & 44 & -54 & 56 & 4.94 \\
\hline Lateral parietal, left & -40 & -62 & 54 & 5.71 \\
\hline
\end{tabular}

the FPCN and the DAN. These results suggest that during internal cognition, dopamine not only strengthens the relationship between the FPCN and the DMN but also helps to disengage the FPCN from the DAN.

Dopamine has long been proposed to play a role in the modulation of attention. Initial evidence came from observations of patients with dopaminergic disorders. Parkinson's disease patients are particularly impaired at shifting between mental sets, as shown by performance on set-shifting tasks like the Wisconsin card sorting test (Lees and Smith, 1983). Treatments for attention deficit hyperactivity disorder, such as methylphenidate and amphetamine, target the dopamine system (Solanto, 1998). Additional support comes from animal lesion studies and neuroimaging of humans. Lesioning of dopaminergic terminals in the prefrontal cortex of primates impairs the animal's ability to acquire attentional sets (Crofts et al., 2001), and PET shows dopamine release in humans during set-shifting (Monchi et al., 2006).

Dopamine may modulate attention by influencing the properties of large-scale, interregional networks (Walters et al., 2000). Local field potential recordings found that dopamine modulates brain oscillations in frequencies thought to support perception and cognition (Ward, 2003). Dopamine agonists alter temporal coherence seen in fMRI BOLD fluctuations (Kelly et al., 2009), and dopamine depletion reduces this coherence and its relationship to cognitive performance (Nagano-Saito et al., 2008). Gordon and colleagues (2011) recently reported an association between network coupling and dopamine, using a polymorphism affecting expression of dopamine transporters as a marker of dopamine function. The present finding of an association between dopamine and cross-network couplings between the DAN, DMN, and FPCN are consistent with the proposed effects of dopamine on network properties.

Enhanced FPCN-DMN coupling in the resting state has been hypothesized to support internally guided attention (Smallwood et al., 2012). Evidence for this hypothesis comes from Spreng and colleagues' (2010) finding of increased connectivity between the FPCN and the DMN during an autobiographical planning task. However, in a study using neuropharmacology, negative coupling between the FPCN and the DMN in the resting state was associated with drug-related
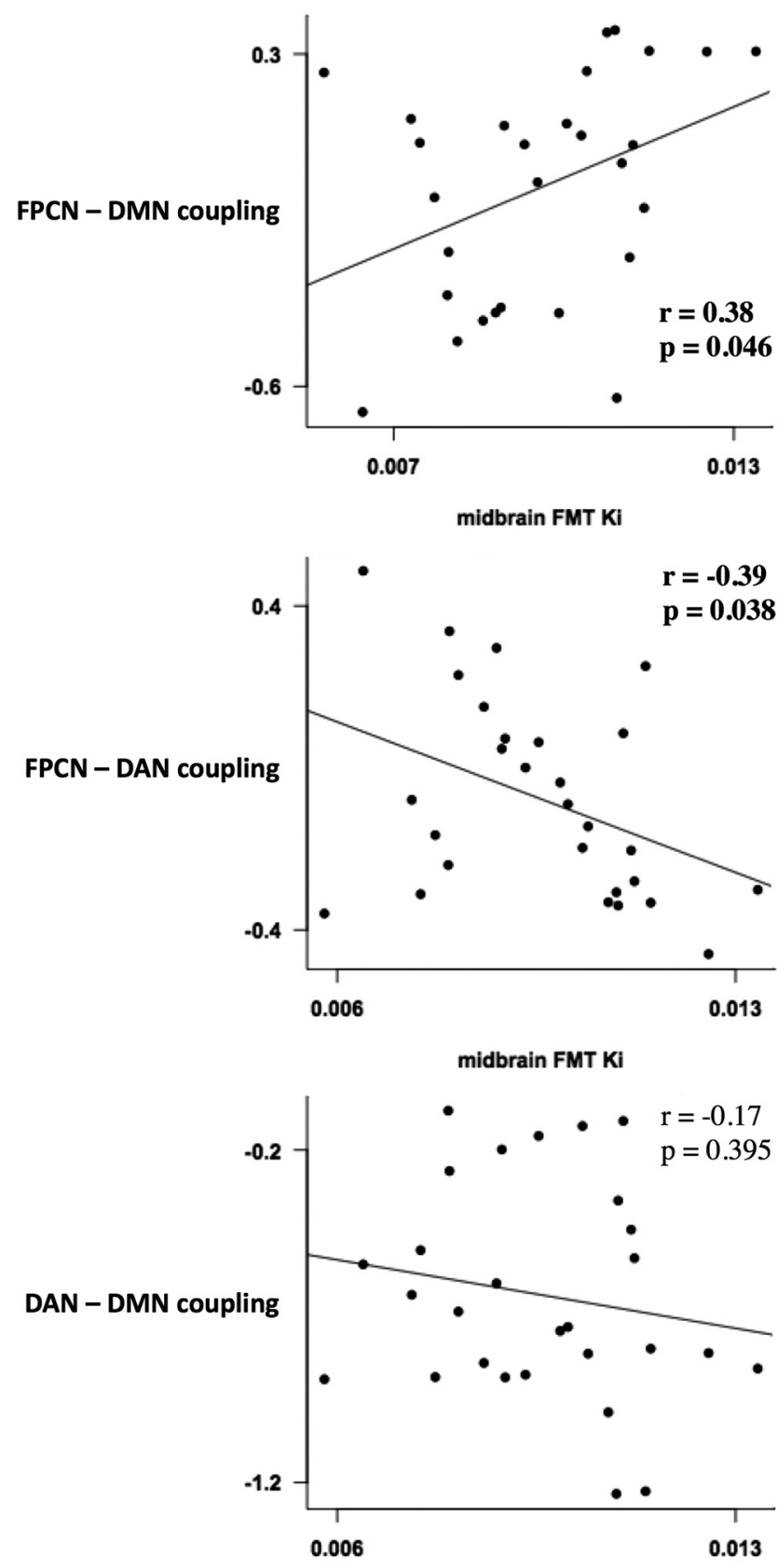

midbrain FMT Ki

Figure 3. Dopamine and network coupling. FMT uptake in the midbrain correlated positively with coupling between the FPCN and DMN (top) and negatively with coupling between the FPCN and the DAN (middle). No relationship found between FMT uptake and coupling between the DAN and the DMN (bottom).

cognitive improvements (Cole et al., 2010). Significant methodological differences exist between these studies, thus complicating interpretation across studies and requiring additional research to resolve these differences.

An alternative interpretation of network coupling also deserves attention. Relationships between dopamine and neural activity or cognitive performance follow an inverted U-shaped profile, with too little or too much dopamine resulting in worse outcomes. Psychopharmacological studies revealed that administration of amphetamine, a dopamine agonist, improved performance in individuals with low endogenous dopamine level but impaired performance in those with high 
endogenous dopamine level (Mattay et al., 2003). Similarly, bromocriptine, another dopamine agonist, interacted with the baseline working memory capacity of subjects, such that the drug improved working memory in subjects with a low baseline performance but impaired working memory in subjects with a high baseline (Kimberg et al., 1997). Furthermore, components of the FPCN also exhibit a U-shaped relationship to task performance (Dumontheil et al., 2010). The U-shaped profile of dopamine and FPCN function suggests that future investigation into the significance of network coupling should consider possible nonlinear effects.

In summary, the current study adds a neurochemical perspective to previous observations of the interaction between the DAN, DMN, and FPCN by showing that, in the absence of external stimulation, dopamine enhances coupling between the FPCN and DMN while reducing the coupling between the FPCN and the DAN. Future research should explore the effect of network coupling in cognition and the role of other neurotransmitter systems in this relationship.

\section{Notes}

Supplemental material for this article is available at http://jagustlab. neuro.berkeley.edu/suppdata.html. The supplemental file shows Pearson's correlations between the time series of each node with every other node in the three networks. One row corresponds to one subject. Sheet 2 explains the column header. For example, a column with the header "fpcn-mpfc-dmn-pcc" shows correlations between the FPCN medial prefrontal node and the DMN posterior cingulate node. This material has not been peer reviewed.

\section{References}

Braskie MN, Wilcox CE, Landau SM, O’Neil JP, Baker SL, Madison CM, Kluth JT, Jagust WJ (2008) Relationship of striatal dopamine synthesis capacity to age and cognition. J Neurosci 28:14320-14328.

Braskie MN, Landau SM, Wilcox CE, Taylor SD, O’Neil JP, Baker SL, Madison CM, Jagust WJ (2011) Correlations of striatal dopamine synthesis with default network deactivations during working memory in younger adults. Hum Brain Mapp 32:947-961.

Cavero I, Massingham R, Lefèvre-Borg F (1982) Peripheral dopamine receptors, potential targets for a new class of antihypertensive agents. Part I. Subclassification and functional description. Life Sci 31:939-948.

Clark BJ, Menninger K (1980) Peripheral dopamine receptors. Circ Res 46:I59-I63.

Cole DM, Beckmann CF, Long CJ, Matthews PM, Durcan MJ, Beaver JD (2010) Nicotine replacement in abstinent smokers improves cognitive withdrawal symptoms with modulation of resting brain network dynamics. Neuroimage 52:590-599.

Corbetta M, Shulman GL (2002) Control of goal-directed and stimulusdriven attention in the brain. Nat Rev Neurosci 3:201-215.

Corbetta M, Kincade JM, Shulman GL (2002) Neural systems for visual orienting and their relationships to spatial working memory. J Cogn Neurosci 14:508-523.

Crofts HS, Dalley JW, Collins P, Van Denderen JC, Everitt BJ, Robbins TW, Roberts AC (2001) Differential effects of 6-OHDA lesions of the frontal cortex and caudate nucleus on the ability to acquire an attentional set. Cereb Cortex 11:1015-1026.

Dumontheil I, Gilbert SJ, Frith CD, Burgess PW (2010) Recruitment of lateral rostral prefrontal cortex in spontaneous and task-related thoughts. Q J Exp Psychol (Hove) 63:1740-1756.

Folstein MF, Folstein SE, McHugh PR (1975) "Mini-mental state" : a practical method for grading the cognitive state of patients for the clinician. J Psychiatr Res 12:189-198.

Fox MD, Snyder AZ, Vincent JL, Corbetta M, Van Essen DC, Raichle ME (2005) The human brain is intrinsically organized into dynamic, anticorrelated functional networks. Proc Natl Acad Sci U S A 102:9673-9678.

Gordon EM, Stollstorff M, Devaney JM, Bean S, Vaidya CJ (2011) Effect of dopamine transporter genotype on intrinsic functional connectivity depends on cognitive state. Cereb Cortex. Advance online publication. Retrieved June 6, 2012. doi: 10.1093/cercor/bhr305.
Greicius MD, Krasnow B, Reiss AL, Menon V (2003) Functional connectivity in the resting brain: a network analysis of the default mode hypothesis. Proc Natl Acad Sci U S A 100:253-258.

Jenkinson M, Bannister P, Brady M, Smith S (2002) Improved optimization for the robust and accurate linear registration and motion correction of brain images. Neuroimage 17:825-841.

Jordan S, Eberling JL, Bankiewicz KS, Rosenberg D, Coxson PG, VanBrocklin HF, O’Neil JP, Emborg ME, Jagust WJ (1997) 6- $\left[{ }^{18} \mathrm{~F}\right]$ fluoro-L-m-tyrosine: metabolism, positron emission tomography kinetics, and 1-methyl-4-phenyl-1,2,3,6-tetrahydropyridine lesions in primates. Brain Res 750:264-276.

Kelly C, de Zubicaray G, Di Martino A, Copland DA, Reiss PT, Klein DF, Castellanos FX, Milham MP, McMahon K (2009) L-dopa modulates functional connectivity in striatal cognitive and motor networks: a double-blind placebo-controlled study. J Neurosci 29:7364-7378.

Kimberg DY, D'Esposito M, Farah MJ (1997) Effects of bromocriptine on human subjects depend on working memory capacity. Neuroreport 8:3581-3585.

Kohli JD (1990) Peripheral dopamine receptors. Am J Hypertens 3:25S-28S.

Kroger JK, Sabb FW, Fales CL, Bookheimer SY, Cohen MS, Holyoak KJ (2002) Recruitment of anterior dorsolateral prefrontal cortex in human reasoning: a parametric study of relational complexity. Cereb Cortex 12:477-485.

Lees AJ, Smith E (1983) Cognitive deficits in the early stages of Parkinson's disease. Brain 106:257-270.

Mattay VS, Goldberg TE, Fera F, Hariri AR, Tessitore A, Egan MF, Kolachana B, Callicott JH, Weinberger DR (2003) Catechol O-methyltransferase val158-met genotype and individual variation in the brain response to amphetamine. Proc Natl Acad Sci U S A 100:6186-6191.

McKiernan KA, Kaufman JN, Kucera-Thompson J, Binder JR (2003) A parametric manipulation of factors affecting task-induced deactivation in functional neuroimaging. J Cogn Neurosci 15:394-408.

Monchi O, Ko JH, Strafella AP (2006) Striatal dopamine release during performance of executive functions: A [(11)C] raclopride PET study. Neuroimage 33:907-912.

Müller U, Suckling J, Zelaya F, Honey G, Faessel H, Williams SC, Routledge C, Brown J, Robbins TW, Bullmore ET (2005) Plasma level-dependent effects of methylphenidate on task-related functional magnetic resonance imaging signal changes. Psychopharmacology (Berl) 180:624-633.

Nagano-Saito A, Leyton M, Monchi O, Goldberg YK, He Y, Dagher A (2008) Dopamine depletion impairs frontostriatal functional connectivity during a set-shifting task. J Neurosci 28:3697-3706.

Nagano-Saito A, Liu J, Doyon J, Dagher A (2009) Dopamine modulates default mode network deactivation in elderly individuals during the Tower of London task. Neurosci Lett 458:1-5.

Patlak CS, Blasberg RG (1985) Graphical evaluation of blood-to-brain transfer constants from multiple-time uptake data: generalizations. J Cereb Blood Flow Metab 5:584-590.

Ptak R, Schnider A (2010) The dorsal attention network mediates orienting toward behaviorally relevant stimuli in spatial neglect. J Neurosci 30:12557-12565.

Raichle ME, Snyder AZ (2007) A default mode of brain function: a brief history of an evolving idea. Neuroimage 37:1083-1090; discussion 1097-1099.

Raichle ME, MacLeod AM, Snyder AZ, Powers WJ, Gusnard DA, Shulman GL (2001) A default mode of brain function. Proc Natl Acad Sci U S A 98:676-682.

Smallwood J, Brown K, Baird B, Schooler JW (2012) Cooperation between the default mode network and the frontal-parietal network in the production of an internal train of thought. Brain Res 1428:60-70.

Smith SM (2002) Fast robust automated brain extraction. Hum Brain Mapp 17:143-155.

Solanto MV (1998) Neuropsychopharmacological mechanisms of stimulant drug action in attention-deficit hyperactivity disorder: a review and integration. Behav Brain Res 94:127-152.

Spreng RN, Stevens WD, Chamberlain JP, Gilmore AW, Schacter DL (2010) Default network activity, coupled with the frontoparietal control network, supports goal-directed cognition. Neuroimage 53:303-317.

Tan HY, Chen Q, Goldberg TE, Mattay VS, Meyer-Lindenberg A, Weinberger DR, Callicott JH (2007) Catechol-O-methyltransferase Val158Met modulation of prefrontal-parietal-striatal brain systems during arithme- 
tic and temporal transformations in working memory. J Neurosci 27:13393-13401.

Tomasi D, Volkow ND, Wang GJ, Wang R, Telang F, Caparelli EC, Wong C, Jayne M, Fowler JS (2011) Methylphenidate enhances brain activation and deactivation responses to visual attention and working memory tasks in healthy controls. Neuroimage 54:3101-3110.

VanBrocklin HF, Blagoev M, Hoepping A, O’Neil JP, Klose M, Schubiger PA, Ametamey S (2004) A new precursor for the preparation of 6$\left[{ }^{18} \mathrm{~F}\right]$ Fluoro-L-m-tyrosine $\left(\left[{ }^{18} \mathrm{~F}\right] \mathrm{FMT}\right)$ : efficient synthesis and comparison of radiolabeling. Appl Radiat Isot 61:1289-1294.

van den Heuvel OA, Groenewegen HJ, Barkhof F, Lazeron RH, van Dyck R, Veltman DJ (2003) Frontostriatal system in planning complexity: a parametric functional magnetic resonance version of Tower of London task. Neuroimage 18:367-374

Vincent JL, Kahn I, Snyder AZ, Raichle ME, Buckner RL (2008) Evidence for a frontoparietal control system revealed by intrinsic functional connectivity. J Neurophysiol 100:3328-3342.

Wager TD, Jonides J, Reading S (2004) Neuroimaging studies of shifting attention: a meta-analysis. Neuroimage 22:1679-1693.

Walters JR, Ruskin DN, Allers KA, Bergstrom DA (2000) Pre- and postsynaptic aspects of dopamine-mediated transmission. Trends Neurosci 23:S41-S47.

Ward LM (2003) Synchronous neural oscillations and cognitive processes. Trends Cogn Sci 7:553-559. 